\title{
Análise descritiva da satisfação dos alunos usuários do restaurante universitário, da Universidade Federal Rural da Amazônia, Belém, Pará
}

\author{
Descriptive analysis of the satisfaction of student users of the university restaurant, Universidade \\ Federal Rural da Amazônia, Belém, Pará
}

Análisis descriptivo de la satisfacción de estudiantes usuarios del restaurante universitario, Universidade Federal Rural da Amazônia, Belém, Pará

\author{
Alexandro Monteiro de Jesus \\ ORCID: https://orcid.org/0000-0002-3299-8325 \\ Universidade Federal da Amazônia, Brasil \\ E-mail: alexandro420dejesus@gmail.com \\ Adriano Joaquim Neves de Souza \\ ORCID: https://orcid.org/0000-0001-6512-5830 \\ Universidade Federal Rural da Amazônia, Brasil \\ E-mail: ad.age13@gmail.com \\ Glinda Sâmia da Silva Fôro \\ ORCID: https://orcid.org/0000-0003-1782-8923 \\ Universidade Federal Rural da Amazônia, Brasil \\ E-mail: glindaforo@gmail.com \\ Gabriel Monteiro de Jesus \\ ORCID: https://orcid.org/0000-0003-0957-3254 \\ Universidade Federal Rural da Amazônia, Brasil \\ E-mail: gabriel200ufra@gmail.com \\ Maria de Lourdes Souza Santos \\ ORCID: https://orcid.org/0000-0003-2797-6014 \\ Universidade Federal Rural da Amazônia, Brasil \\ E-mail: mdelssantos@yahoo.com.br
}

\begin{abstract}
Resumo
O restaurante universitário (RU) da UFRA, atende a servidores públicos, discentes, e outros, desde que tenham vínculo com a universidade, ofertando refeições no horário de almoço, para os discentes a refeição é gratuita, que aliado à procura de refeições balanceadas e de qualidade, ocasiona uma maior demanda de usuários. Logo esse estudo visa avaliar a percepção dos discentes usuários em relação aos serviços prestados pelo Restaurante Universitário. Para tal foi realizada uma pesquisa do tipo survey, por intermédio de questionários estruturados a partir de afirmações com escala do tipo Likert de 5 pontos. O questionário foi aplicado a 230 discentes usuários do RU, que aceitaram em participar da pesquisa, durante o horário de atendimento para o almoço, na fila de entrada. Os dados adquiridos foram analisados com o software Microsoft Excel, versão 2016. Os resultados mostram insatisfação quanto ao tempo de espera, relacionado a dimensão atendimento e o melhor avaliado satisfatoriamente foi o da qualidade do complemento servida junto com o prato principal, enquadrando-se na dimensão, refeição (alimento).
\end{abstract}

Palavras-chave: Refeição; Estatística descritiva; Perfil de usuários.

\begin{abstract}
The university restaurant (RU) of UFRA serves public servants, students, and others, as long as they have a connection with the university, offering meals during lunch hours, for students the meal is free, which combined with the search for balanced and quality meals, causes greater user demand. Therefore, this study aims to assess the perception of student users in relation to the services provided by the University Restaurant. To this end, a surveytype survey was conducted, using questionnaires structured from statements with a 5-point Likert scale. The questionnaire was applied to 230 student users in the UK, who agreed to participate in the survey, during lunch hours, in the entrance queue. The acquired data were analyzed using Microsoft Excel software, version 2016. The results show dissatisfaction regarding the waiting time, related to the service dimension and the best evaluated satisfactorily was the quality of the complement served along with the main dish, fitting in the dimension, meal (food).
\end{abstract}

Keywords: Meal; Descriptive statistics; User profile. 


\begin{abstract}
Resumen
El restaurante Universitario (RU) de la UFRA atiende a servidores públicos, estudiantes y otras personas, siempre que estén vinculadas a la universidade, ofreciendo comidas a la hora del almuerzo, para los estudiantes la comida es gratuita, lo que combinado con la búsqueda de comidas equilibradas y la de calidad, provoca una mayor demanda por parte de los usuarios. Por tanto, este estudio tiene como objetivo evaluar la percecpción de los estudiantes usuarios en relación a los servicios que oresta el Restaurante Universitario. Para ello, se realizó una investigación tipo encuesta, mediante cuestionarios estructurados basados en enunciados con una escala tipo Likert de 5 pontos. El cuestionario se aplicó a 230 estudiantes usuarios del Reino Unido, que aceptaron participar en la encuesta, durante las horas del almuerzo, en la línea de entrada. Los datos adquiridos fueron analizados mediante el software Microsoft Excel, versión 2016. Los resultados muestran insatisfacción con el tiempo de espera, relacionado con la dimensión de servicio, y la mejor valorada satisfactoriamente fue la calidad del complemento servido con el plato principal, en consonancia con la dimensión, comida.
\end{abstract}

Palabras clave: Comida; Estadísticas descriptivas; Perfil del usuario.

\title{
1. Introdução
}

O comportamento dos usuários de serviços de restaurantes universitários se modificou, deixando de lado o lazer e a diversão atrelados aos restaurantes universitários (RU's) para uma atividade mais relacionada a necessidade, pois os estudantes universitários apresentam uma carga horária extensa e ou moram longe da Universidade e não tem tempo para preparar suas próprias refeições, com isso optam por realizarem suas refeições nos RU's das Universidades, contribuindo para o aumento da demanda da atividade dos restaurantes universitários (Rebelato, 1997; Passador et al., 2006; Saraiva, 2016).

Esse aumento da demanda de usuários de $\mathrm{Ru}$ é devido também a adesão das universidades federais, por meio do Decreto $\mathrm{n}^{\circ} 6.096$ de 24 de abril de 2007, ao Programa de Apoio ao Plano de Reestruturação das Universidades Federais (REUNI), que consiste principalmente no aumento da quantidade de cursos, maiores disponibilidades de vagas por cursos de graduação e no combate da evasão de universitários, dentre outras medidas adotadas (Oliveira, 2013).

Com isso os restaurantes universitários são tidos como importante ferramenta de assistência estudantil, pois fornecem, uma opção de alimentação, principalmente para aqueles alunos com alguma vulnerabilidade financeira e ou que precisam se deslocar longas distâncias para chegar na Universidade, desse modo o RU é um dos tipos importantes de alimentações realizadas coletivamente, que são Unidades de Alimentação e Nutrição (UAN's) (Rohr et al., 2010).

As Unidades de Alimentação e Nutrição (UAN's) são responsáveis pela produção de refeições que respeitem os padrões higiênico-sanitários recomendados, que sejam balanceadas e que tenham boas características organolépticas, além de satisfazer o usuário quanto ao serviço prestado (Abreu; Spinelli, 2009; Colares; Freitas, 2007; Oliveira, 2004; Proença et al., 2005; Proença, 1999).

No trabalho de Vieira e Cavalcanti (2020) realizado nos refeitórios das instituições públicas da cidade Campina grande -Paraíba (2020) afirmam que a qualidade dos alimentos possui conceitos variados e dinâmicos, mas fundamentalmente indica o nível de excelência daquilo que é produzido (produto ou serviço). No âmbito dos serviços, a qualidade está ligada a satisfação dos consumidores, e representa um conceito vital para as organizações que atuam nessa categoria.

Diante disso, os RU's necessitam dispor de um cardápio qualitativa e quantitativamente diversificado e equilibrado com o objetivo de favorecer a saúde de seus usuários e para tal situação é recomendável também atenção para as qualidades sensoriais e higiênico-sanitárias, assim como um bom atendimento, atendendo os funcionários e os alunos da universidade (Maia, 2008; Oliveira \& Alves, 2008; Brandão \& Giovanoni, 2011).

Desta maneira, há outros fatores além da comida que auxiliam para uma boa experiência de consumo em restaurantes, como por exemplo as questões das expectativas subjetivas e fisiológicas dos usuários. Com isso, é necessário melhorar a qualidade dos serviços prestados (Domingues, 2012).

No entanto, há na maioria das vezes diferenças na visão de expectativa do usuário em detrimento da percepção da administração do serviço oferecido. De modo geral o objetivo primordial da visão da qualidade de RU's era direcionada 
geralmente em oferecer refeições adequadas e saudáveis, entretanto outras questões foram adicionadas no serviço dos restaurantes (Parasuraman et al., 1985). Por mais que a opinião a respeito da percepção de um serviço oferecido seja diferente para cada pessoa, o administrador necessita saber o que os usuários esperam do serviço, para a partir desse ponto traçar planos de melhorias de performance que podem angariar noções positivas (Reis, 2001).

Os ambientes que são servidas as refeições aos acadêmicos devem fornecer alimentos com adequada qualidade higiênico-sanitária, conter valor nutricional e sensorial, além de realizar ações de educação alimentar e nutricional, incentivando hábitos saudáveis (Lira, et., 2020).

Logo a importância de realização de avaliações periódicas com pesquisas de satisfação dos usuários quanto as refeições ofertadas, tendo dessa maneira informações atualizadas do serviço prestado (Proença, Souza, Hering, 2005). A satisfação seria a reação e ou sentimento a uma expectativa do produto ou serviço consumido de determinado fornecedor (Solomon, 2002).

A satisfação pode ser entendida como sendo uma resposta ao grau de contentamento do consumidor, em que ele identificará e determinará qual ou quais características de um produto ou serviço que está sendo oferecido, se relacionará com um grau de contentamento prazeroso, para mais ou para menos (Oliver, 1997), ou seja, satisfação está ligada à saciedade do consumidor que espera conseguir, ao adquirir o produto e ou serviço, de modo a que conscientemente ou inconscientemente atribuem valores as disponibilidades de consumo (Longart, 2015), o entendimento da satisfação do consumidor favorece o gerenciamento e orientação para a indústria (Dipietro \& Levitt, 2017).

A pesquisa de satisfação é uma ferramenta necessária para a obtenção de informações atuais e importantes em relação das necessidades dos clientes (usuários do RU, neste caso), criando padrões mais adequados de atendimento, de acordo com seu cliente, ganhar a fidelização, maximizar a satisfação e diminuir ou anular a insatisfação (Rossi \& Slongo, 1998). Para tal, coletar opiniões, críticas e sugestões, fato crucial para as empresas, pois saber ouvir os clientes indicam a necessidade de alterações e melhoramento de produtos e ou serviços ofertados, dado que o atual mercado consumidor vai além da produção de bens e serviços, atendendo a satisfação dos clientes ao saber suas necessidades (Trierweiller et al., 2011), perguntando o que os clientes querem, precisam ou esperam e saber como avaliam os bens e serviços (Milet, 1997).

A adesão do REUNI, como visto anteriormente, promoveu um aumento de alunos e servidores nas Universidades Federais. Entretanto, as Universidades não foram preparadas para atender esse aumento da demanda, resultado disso é a falta de estrutura física, econômica e de efetivo pessoal, desse modo, pode afetar a satisfação dos usuários (Maia, 2008; Marques et al., 2010; Oliveira, 2013).

Com isso, esse trabalho tem como objetivo identificar e avaliar o grau de satisfação dos alunos que usam os serviços prestados pelo Restaurante Universitário da Universidade Federal Rural da Amazônia - UFRA.

\section{Metodologia}

A pesquisa foi realizada no Restaurante Universitário na Universidade Federal Rural da Amazônia (UFRA), no campus sede em Belém-Pará, onde são servidas diariamente em média 600 refeições para alunos de graduação, pós-graduação, e servidores, sendo que para os alunos de graduação a refeição é oferecida gratuitamente e é direcionada a maioria das refeições, 500 no total.

A pesquisa foi realizada pelo método misto, composta de duas abordagens a qualitativa e quantitativa, sendo a primeira uma pesquisa exploratória, objetivando identificar as questões mais relevantes em Restaurantes Universitários, tendo desse modo um foco no estudo, a segunda etapa caracteriza-se mais como descritiva pois pretende identificar e mensurar o grau de satisfação, para descrever as expectativas, percepção, sugestões e ou críticas dos usuários do Restaurante Universitário da UFRA. 
O survey foi empregado na pesquisa direcionado para os alunos de graduação, por conta de serem a maioria dos usuários, sendo entrevistados 230 alunos usuários do RU, que concordaram em participar, e devolveram o questionário completamente e corretamente preenchido. Para tal, os usuários foram abordados aleatoriamente na fila de entrada para o restaurante, começando no horário das 11:00h até 12:30h, iniciando no dia dezesseis de maio de 2019 até o dia vinte e cinco de maio de 2019, buscando abranger de maneira satisfatória os estudantes, no primeiro momento foi realizada a identificação dos pesquisadores e posteriormente explicado a finalidade da pesquisa.

Os dados foram digitalizados e analisados no programa Excel, versão 2016, sendo empregados a análise descritiva como médias, desvios padrões etc.

Etapa qualitativa

A pesquisa qualitativa é a primeira etapa a ser tomada, quando se trata de estudar a satisfação sobre determinados assuntos, e que ainda não têm direcionamento de ideias para abordar o assunto objeto da pesquisa (Motta, 1999). Busca estudar uma realidade que não pode ser mensurada, atuando nas categorias de significados, motivos, aspirações, crenças etc., que são relações e processos que não podem ser minimizados em variáveis operacionais (Minayo, 1994), e além disso segundo Santos e Vera (2021) retratam a importância de utilizar-se a pesquisa quantitativa tem como intuito quantificar os dados, a qual vai possibilitar um tratamento estatístico para melhor compreensão sobre as respostas dos discentes sobre as refeições que são oferecidas pela instituição.

Logo esta etapa é fundamental no início da pesquisa, pois nesta etapa o conhecimento e compreensão sobre o objeto de estudo ainda é mínimo, por se deter a explorar (exploratória), busca conhecer as variadas formas de explicações para acontecimentos relacionados com o marketing (Mattar, 2001, p.18).

Para tal, realizou-se pesquisas de satisfação empregadas em outros RU's de distintas Universidades brasileiras. Além de que foi utilizado o método de observação do tipo direta e oculta, tendo a participação efetiva e presencial do pesquisador, sendo de desconhecimento dos alunos que frequentam o RU, para que se evitasse respostas tendenciosas e ou de descontentamento com a gestão Universitária, de cunho política, e para o conhecimento dos assuntos que norteiam o funcionamento do RU, de acordo com a sua satisfação, em uma conversa franca, honesta e informal realizado de forma imparcial.

Dessa forma, permitiu uma melhor abordagem da etapa seguinte que é a quantitativa, pois auxiliou a determinar as variáveis a serem abordadas, predizendo quais inserir em um questionário para a mensuração da satisfação dos usuários (Motta, 1999), fornecendo informações que complementem ou fortaleçam a etapa de pesquisa de satisfação em outros Restaurantes Universitários.

Etapa quantitativa

A pesquisa quantitativa comporta-se como descritiva, caracterizando-se por apresentar direcionamento da pesquisa bem definido, bem estruturada, utiliza procedimentos formais e é voltada para a solução de problemas, por isso é tida como conclusiva buscando explicar a natureza e as características próprias dos fatos organizacionais e sociais, descrevendo as características das categorias importantes, mensurar a porcentagem dos comportamentos específicos das unidades do estudo, analisar as percepções de características dos produtos e medir o nível de associação das variáveis e estimar previsões inerentes para cada objeto de estudo precisando-se de grande conhecimento à respeito (Malhotra, 2001; Mattar, 2001).

Esta etapa foi predominante na pesquisa e que o questionário foi o principal instrumento da coleta de dados, permitindo informações mais homogêneas, permitindo uma confiança mais apurada dos dados e das tomadas de decisões (Motta, 1999).

Com isso foi possível mensurar o grau de satisfação dos alunos usuários da UFRA, em relação aos serviços prestados pelo restaurante universitário. 
Elaboração do questionário

O questionário feito para essa pesquisa, é dividido em quatro partes e uma pergunta aberta em que o respondente colocaria sua crítica, sugestão e elogios. Na primeira seção buscou-se traçar o perfil do usuário que frequentava o Restaurante Universitário. Na segunda parte pretendeu-se conhecer a avaliação em relação ao atendimento prestado pelos colaboradores do RU, na terceira e na quarta pretendeu-se conhecer a percepção do discente quanto a estrutura física do RU, e saber a percepção quanto a avaliação da qualidade dos alimentos oferecidos. A avaliação da percepção do usuário ocorreu em escalas Linkert oriundo da conversa dos autores com os discentes da instituição e da pesquisa realizados na etapa qualitativa, em que o aluno tinha que julgar uma afirmativa pertinente ao RU.

A escala foi dividida em cinco pontos, sendo o número um, o discente "não concordava" com a afirmativa e quando escolhia o número cinco representa o "concordo totalmente" com a afirmativa, a escala dividida em cinco pontos fornece uma pontuação de quebra definido entre a satisfação e a insatisfação, por ser um número ímpar fornece a mesma quantidade de categorias favoráveis e desfavoráveis e um meio termo sendo central e neutro, representado pela escala 3 , sendo os resultados obtidos a reflexão do quanto há diferenças entre as respostas nos questionários relacionadas aos atributos abordados, e posteriormente comparar as diferenças entre as variáveis (Rossi e Slongo, 1998). As escalas utilizadas foram: 1- Não concordo, 2- Não concordo parcialmente, 3-Indiferente, 4- Concordo parcialmente, 5-Concordo totalmente. Sendo os números 1 e 2 relacionados a insatisfação, o 3 a imparcialidade, e o 4 e 5 relacionados a satisfação.

\section{Resultados e Discussão}

\section{Caracterização dos alunos}

Em relação a questão perfil social e acadêmico dos participantes obteve-se os seguintes resultados, quanto ao gênero a maioria correspondia ao gênero feminino com $57 \%$ do total e $43 \%$ do gênero masculino. Em relação a idade, a amplitude foi de 17 a 40 anos, com média de 21,6 anos, sendo a faixa etária de maior representatividade a de 17 a 25 anos, sendo 83,9\% da amostra, notando-se uma parcela de usuários jovens do RU. Quanto o estado civil 93,5\% dos entrevistados se declararam solteiros seguidos de união estável com $3,9 \%$, casado com $2,2 \%$ e divorciado com $0,4 \%$.

No que condiz ao perfil acadêmico, os alunos dos cursos, Agronomia e Engenharia de Pesca apresentaram os maiores valores porcentuais $26,52 \%$ e $24,35 \%$, respectivamente dos entrevistados que usam o RU, abrangendo em menor proporção os outros cursos (Figura 1).

Figura 1: Identificação dos cursos dos usuários do RU.

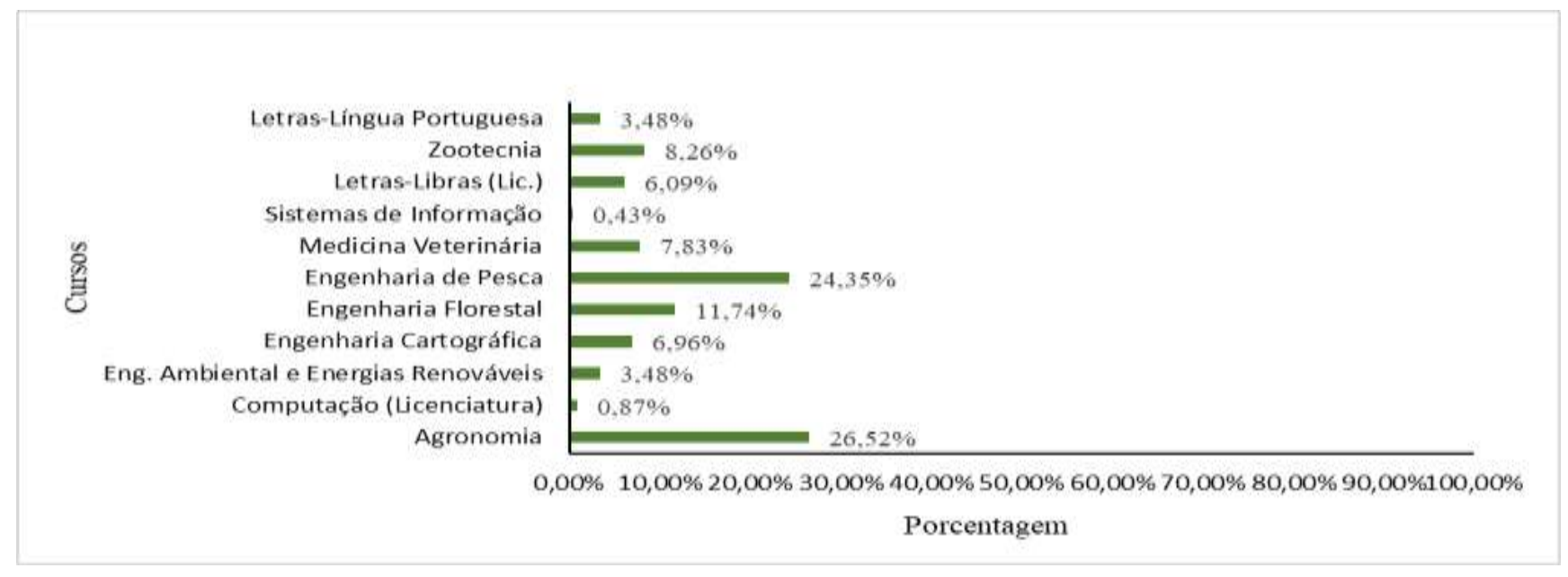

Fonte: Dados da pesquisa. 
Quanto a atividade de estágio, evidenciou-se que a maioria não estagia (59,57\%), seguido dos que estagiam, mas não é remunerado $(22,17 \%)$ e estagiam de forma remunerada $(18,26 \%)$. O período das aulas dos entrevistados que predominou foi o matutino $(81,74 \%)$, posteriormente o vespertino $(16,96 \%)$ e o noturno $(1,3 \%)$, entretanto é importante ressaltar que na UFRA há cursos de período integral, como o de Agronomia.

No que concerne à frequência, $62,17 \%$ dos usuários frequentam o RU diariamente para almoçarem, 24,78\% frequentam de três a quatro vezes por semana, 10,0\% duas vezes por semana, e 3,04\% uma vez na semana, demonstrando que no RU da UFRA, 86,95\% dos alunos, frequentam pelo menos três vezes na semana o RU, esses resultados corroboram com outro levantamento, em que 86,1\% dos usuários utilizam o RU pelo menos três vezes na semana (Cattafesta et al., 2012). Quanto ao uso diário do RU, os resultados obtidos apresentam certa diferença com outros estudos obtidos, em que somente 27\% dos usuários almoçam diariamente no RU da Universidade Federal do Rio Grande do Norte (UFRN) (Medeiros et al., 2013), e em outro estudo, 44,47\% almoçam diariamente no RU da UFSM, a gratuidade do almoço no RU da UFRA, pode ser considerado como um fator de incentivo para maior uso do RU (Junior et al., 2015).

Satisfação no atendimento

No referente ao atendimento prestado pelos colaboradores do RU, foi abordado as seguintes questões como: atendimento organizado, tempo de espera, oferta de refeições satisfatória para a demanda de alunos, postura ética de atendimento dos funcionários, seus resultados estatísticos se encontram no Quadro 1 que vem a seguir. O valor médio do item postura ética do atendimento foi o único enquadrado de indiferente (neutro), com média de 3,8043 para concordo parcialmente (satisfação em parte), se considerado a mediana de 4, demonstrando boa satisfação dos alunos quanto a esse quesito. A boa satisfação quanto a esse quesito relacionado aos colaboradores, também foi encontrado no RU da Universidade Federal de Santa Maria (UFSM), em que o atendimento, a postura e a higiene dos funcionários, foram avaliadas por 70,17\% dos entrevistados como ponto positivo (Junior et al., 2015), também positivamente avaliado no RU do Campus de Londrina da UTFPR, em que 43,0\% consideram boa ou ótima a cordialidade dos colaboradores.

Enquanto a eficiência e organização do atendimento média obtida foi de 2,7522, podendo-se enquadrar como discordo em parte, e com mediana 2, fato que evidencia certa insatisfação quanto a esse item avaliado, o que pode ser evidenciado também no quesito tempo de espera, que será descrito posteriormente, em desacordo com a pesquisa feita no RU do Campus de Londrina da UTFPR, em 59\% consideram boa ou ótima a pontualidade no atendimento (Gardin \& Cruvinel, 2014), e no RU da UFMT, a média de satisfação ficou em 6,25, considerando em uma escala de 0 a 10 (Hárter et al., 2013).

O tempo de espera na fila e a quantidade de oferta das refeições podem ser classificadas como discordo totalmente e discordo em parte, com as menores médias e medianas, respectivamente, 1,6130, 1 e 1,6739, 1, demonstrando, insatisfação dos alunos, quanto a esses últimos itens, resultado que corrobora com outros estudos como o realizado no RU do campus de Londrina da UTFPR, em que 73,1\% consideram péssimo, ruim ou regular o tempo de espera na fila do RU (Gardin \& Cruvinel, 2014), no RU da UFMT, a nota média de satisfação foi de 4,57, considerando uma escala de 0 a 10 (Hárter et al., 2013) e no RU da UFF 87,2\% consideram insatisfatório o tempo de espera na fila (Carvalho, Amorim \& Tavares, 2003). Esses quesitos mostram que os RU's apresentam certa deficiência para atender a demanda de usuários. 
Quadro 1: Avaliação do atendimento do RU, na UFRA, em 2019.

\begin{tabular}{|l|c|c|c|c|c|c|c|}
\hline \multicolumn{1}{|c|}{ Estatística } & Mínimo & Máximo & Média & Mediana & Desvio padrão & Variância & Erro padrão \\
\hline Informações & 1 & 5 & 2,7522 & 2 & 1,3492 & 1,8204 & 0,0890 \\
\hline 2* tempondimento & 1 & 5 & 1,6130 & 1 & 0,9451 & 0,8933 & 0,0623 \\
\hline 3* oferta & 1 & 5 & 1,6739 & 1 & 1,2823 & 1,6443 & 0,0846 \\
\hline 4*Funcionários & 1 & 5 & 3,8043 & 4 & 1,2117 & 1,4681 & 0,0799 \\
\hline
\end{tabular}

Nota: $1^{*}$ Atendimento é eficiente e organizado; $2^{*} \mathrm{O}$ tempo de espera na fila é tolerável; $3^{*}$ a oferta de refeições é suficiente para os alunos; 4* os funcionários apresentam postura ética no atendimento. Fonte: Dados da pesquisa.

No referente a estrutura do RU, a melhor média $(3,9913)$ foi referente a iluminação enquadrando-se da zona de indiferença e da concordo parcialmente, configurando-se boa iluminação no momento da alimentação, no RU da UFSM, esse item também teve boa satisfação percentual dos usuários com 87,17\% (Junior et al., 2015), seguida respectivamente pelas médias da disposição das mesas do espaço $(3,6957)$, considerado com layout aceitável, corroborando com o trabalho no RU de UFSM, em que 60,33\% estão satisfeitos com layout, considerando a distribuição das mesas e das cadeiras e outros fatores de distribuição física, já no RU da UFPE, somente 30\% consideram satisfatório o arranjo físico

De acordo com Alves e Salcedo (2014) consideram importante a quantidade de lugares e o tamanho nos refeitórios para os discentes almoçarem de forma confortáveis, contudo no RU do Campus de Ponta Grossa da Universidade Tecnológica Federal do Paraná (UTFPR), o espaço físico foi considerado como ruim por 75,75\%, sendo o pior item analisado da pesquisa (Braga et al., 2015), a ventilação com média de $(3,1913)$ e mediana de 4, considerada portanto, da zona de indiferença para concordo parcialmente, terceiro quesito bem avaliado, bem avaliada também no RU da UFMT, com nota média de avaliação de 7,30, em uma escala de 0 a 10 (Hárter et al., 2013), e também foi bem avaliado no RU de Ponta Grossa da UFTPR, em que somente $4 \%$ consideram a ventilação ruim (Braga et al., 2015), diferentemente do encontrado no RU da UFF, em que o grau de insatisfação foi de 52,2\% (Carvalho et al., 2003).

Em relação as medianas (4) esses três itens podem ser considerados como concordo parcialmente (4). Enquanto que o item: quantidade de mesas e cadeiras é suficiente para atender a demanda de alunos apresentou média e mediana de 2,9043, 3 ficando na zona de neutralidade e concordo parcialmente, em relação ao bom estado das cadeiras, a média e mediana obtida foi respectivamente de 2,3217, 2, ficando na zona de concordo em parte, apresentando menos satisfação se comparado com os itens anteriores, fato também que foi evidenciado no RU do Campus Ponta Grossa da UFTPR, em que somente 13,75\% consideram o mobiliário bom ou ótimo (Braga et al., 2015), e no RU do Campus Medianeira da UTFPR, somente menos de 20\% consideram as cadeiras confortáveis (Gasparin et al., 2012), e o item espaço externo fornece proteção, foi o que teve a maior rejeição, com média de 2,2826 (discordo parcialmente) e mediana de 1 (discordo totalmente), ficando os alunos de graduação usuários do RU, suscetíveis ao sol e chuva, como demostrados pelos valores da Quadro 2. 
Quadro 2: Avaliação da infraestrutura do RU, na UFRA, em 2019.

\begin{tabular}{|l|c|c|c|c|c|c|c|}
\hline Informações & Mínimo & Máximo & Média & Mediana & Desvio padrão & Variância & Erro padrão \\
\hline 1*Quantidade & 1 & 5 & 2,9043 & 3 & 1,4141 & 1,9995 & 0,0932 \\
\hline $2 *$ Cadeiras & 1 & 5 & 2,3217 & 2 & 1,2783 & 1,6340 & 0,0843 \\
\hline 3* As mesas & 1 & 5 & 3,6960 & 4 & 1,2923 & 1,6712 & 0,0852 \\
\hline $4 *$ Iluminação & 1 & 5 & 3,9913 & 4 & 1,1821 & 1,3973 & 0,0779 \\
\hline 5*Ventilação & 1 & 5 & 3,1913 & 4 & 1,5063 & 2,2689 & 0,0993 \\
\hline 6* Espaço & 1 & 5 & 2,2816 & 1 & 1,5702 & 2,4626 & 0,1035 \\
\hline
\end{tabular}

Nota: $1 *$ A quantidade de mesas e cadeiras é suficiente; $2 *$ as cadeiras estão em bom estado; $3 *$ As mesas estão bem-dispostas no espaço;4* A iluminação é suficiente; $5^{*}$ A ventilação é suficiente; $6^{*} \mathrm{O}$ espaço externo de espera é protegido. Fonte: Dados da Pesquisa.

Em relação as refeições oferecidas no RU, os itens de maior concordância pelos alunos foram, respectivamente, em relação ao sabor da refeição com média de 4,3, qualidade dos alimentos ofertados, $(4,2565)$, complemento de qualidade $(4,1957)$, e quantidade de proteína oferecida $(4,0217)$, enquadrando-se na concordância parcial. Enquanto a variedade no cardápio semanal e a diversidade do complemento tiveram os menores valores com médias, respectivamente de (39478) e (3,7652), considerado na zona de indiferença e concordância parcial.

As medianas obtidas de todos os itens foram no valor de 4 sendo desse modo, representando a concordância parcial, como mostra o Quadro 3.

O sabor da refeição no Quadro 3, foi o mais bem avaliado de todos os itens desta pesquisa, fato não observado no RU de UFSM, em que se verificou grande índice de insatisfação de $44,81 \%$, relacionados com o sabor e tempero da refeição, no RU da UFPE, já no RU do Campus Londrina da UTFPR, esse índice foi menor com apenas 12,2\% (Gardin \& Cruvinel, 2014), no RU da Universidade Estadual de Campinas (UNICAMP), os usuários (73\%) não consideram que a carne tenha um sabor agradável (Almeida et al., 2008). A qualidade dos alimentos ofertados vem logo em seguida, demonstrando satisfação dos usuários, quanto a refeição de uma forma geral, que também está relacionado com a qualidade dos complementos das refeições, enquadrando-se o arroz, feijão, farofa, salada, verduras, legumes, ou seja, o acompanhamento das refeições oferecidas. No RU da UEL, 44\% estavam satisfeitos com a qualidade das refeições (RU, 2013b), já no RU da UFU, o percentual de usuários satisfeitos é maior, com 71\% (RU, 2014). No RU do Campus Ponta Grossa é quase unanimidade a satisfação em relação a qualidade dos alimentos, com $92,75 \%$ que consideraram boa ou ótima a qualidade dos alimentos (Braga et al., 2015).

A quantidade de proteína ofertada apresentou concordância parcial, a variedade do cardápio semanal e a diversidade apresentaram abrangência da zona de indiferença para a concordo parcialmente, apresentando resultados consideráveis também em outros trabalhos, como no RU do Campus Londrina da UTFPR, que 22\% dos usuários não estavam satisfeitos com a variedade diária (Gardin \& Cruvinel, 2014), no RU do Campus Ponta Grossa da UFTPR, 22,5\% dos usuários consideram ruim a variedade (Braga, Pereira \& Andrade Junior, 2015), e no RU noturno da UFSC, 16\% consideram a variedade ruim (Jacob et al., 2006), e no RU do Campus Ministro Reis Velloso da UFPI, 31,7\% consideram satisfatória a variedade dos pratos servidos (Moura et al., 2015), e 15\% dos usuários estavam insatisfeitos com a variação do cardápio semanal, no RU da UFU (Ru, 2014). 
Quadro 3: Avaliação dos alimentos do RU, na UFRA, em 2019.

\begin{tabular}{|l|c|c|c|c|c|c|c|}
\hline Informação & \multirow{2}{*}{ Mstatística } & Máximo & Média & Mediana & Desvio padrão & Variância & Erro padrão \\
\hline 1*Alimentos & 1 & 5 & 4,2565 & 4 & 0,9619 & 0,9252 & 0,0634 \\
\hline 2* Sabor & 1 & 5 & 4,30 & 4 & 0,9164 & 0,8397 & 0,0604 \\
\hline 3*Quantidade & 1 & 5 & 4,0217 & 4 & 1,1838 & 1,4013 & 0,0781 \\
\hline 4* Variedade & 1 & 5 & 3,9478 & 4 & 1,1124 & 1,2374 & 0,0733 \\
\hline 5*Complemento & 1 & 5 & 4,1957 & 4 & 0,9256 & 0,8567 & 0,0610 \\
\hline 6*Diversificado & 1 & 5 & 3,7652 & 4 & 1,1395 & 1,2983 & 0,0751 \\
\hline
\end{tabular}

Nota: $1 *$ Os alimentos são de boa qualidade; $2 *$ O sabor da refeição é agradável; $3 *$ A quantidade de proteína é suficiente; $4 *$ Há boa variedade do cardápio semanal; $5 * \mathrm{O}$ complemento é de qualidade; $6^{*} \mathrm{O}$ complemento é diversificado.

Satisfação geral dos alunos Fonte: Dados da Pesquisa.

A média geral que obteve o maior valor atribuído pelos alunos foi a relacionada com o atributo sabor da refeição agradável com média de 4,3 considerado de concordo parcialmente e concordo totalmente se destacando, com isso, como melhor item de concordância evidenciado. E o atributo tempo de espera na fila é considerado pelos alunos como o de menor concordância entre todos os itens analisados, com média de 1,6130 (Figura 2).

Figura 2: Grau de satisfação médios atribuídos ao RU.

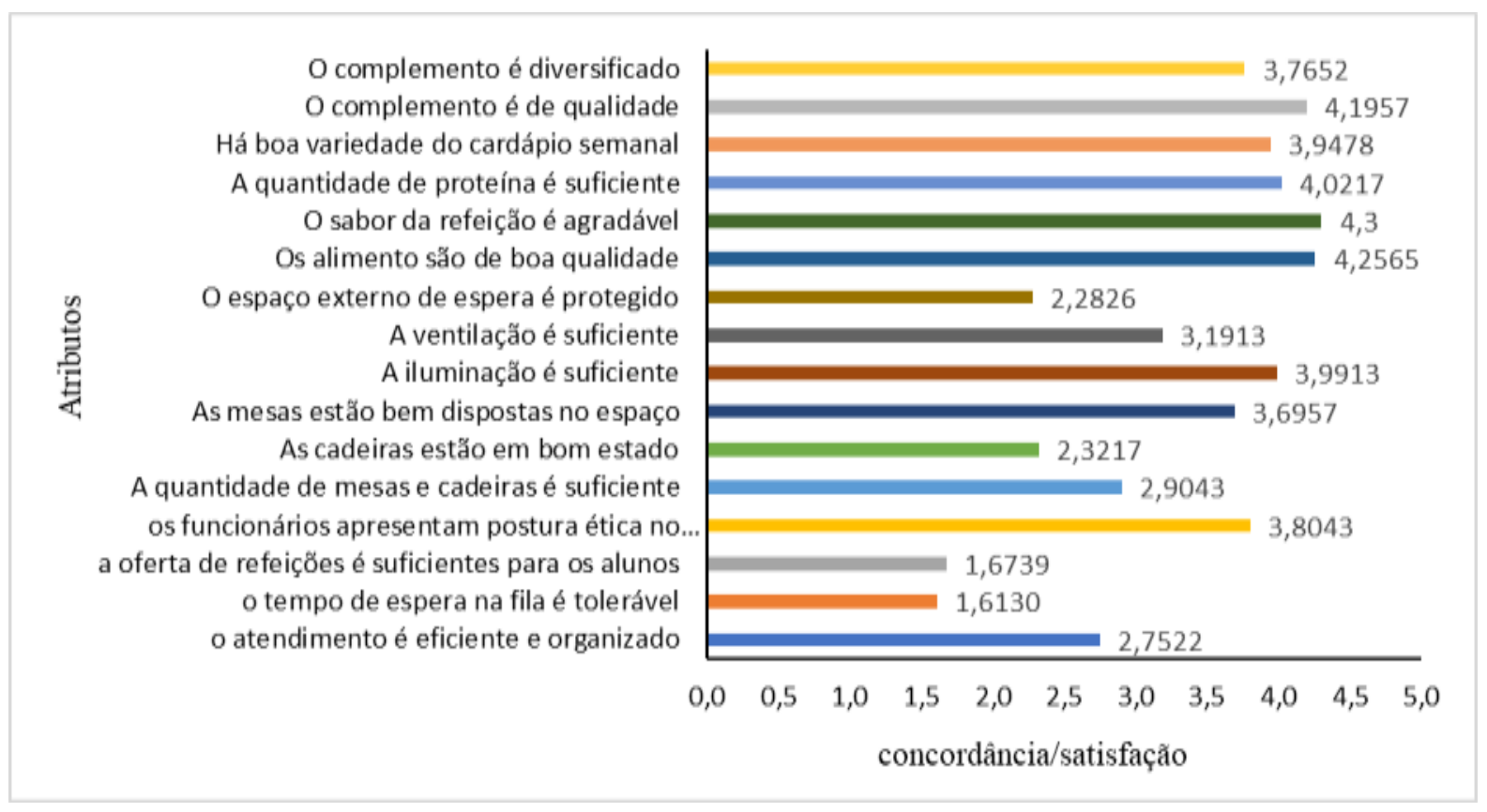

Fonte: Dados da pesquisa.

A divisão em setores dos serviços prestados pelo RU, em atendimento, infraestrutura e qualidade das refeições, permitiu identificar qual setor recebeu uma melhor avaliação na percepção dos alunos usuários, sendo o bloco qualidade das refeições servidas a melhor avaliada, com média total de $(4,0812)$, podendo-se classificada entre concordo parcialmente e concordo plenamente, como está na faixa de 4 e 5, considera-se como atributo satisfatório oferecido pelo RU, seguida da infraestrutura $(3,0645)$ sendo de tal maneira indiferente e o atendimento apresentou o menor valor de avaliação com $(2,4609)$ 
enquadrando-se na faixa de discordo parcialmente e indiferente (Figura 3). Os setores atendimento, infraestrutura e refeições apresentaram grau de avaliação diferentes em outro trabalho, em que os construtos citados apresentaram os maiores valores de satisfação dados pelos usuários do RU da UFMG do campus Pampulha, com média de 7,57 para o atendimento, média de aproximadamente 7,43 para a infraestrutura e média de 7,20 para a qualidade do alimento, ficando entre os três melhores avaliados (Amorim et al., 2019).

Figura 3: Grau de satisfação médios gerais obtidos por setor, atribuídos ao RU.

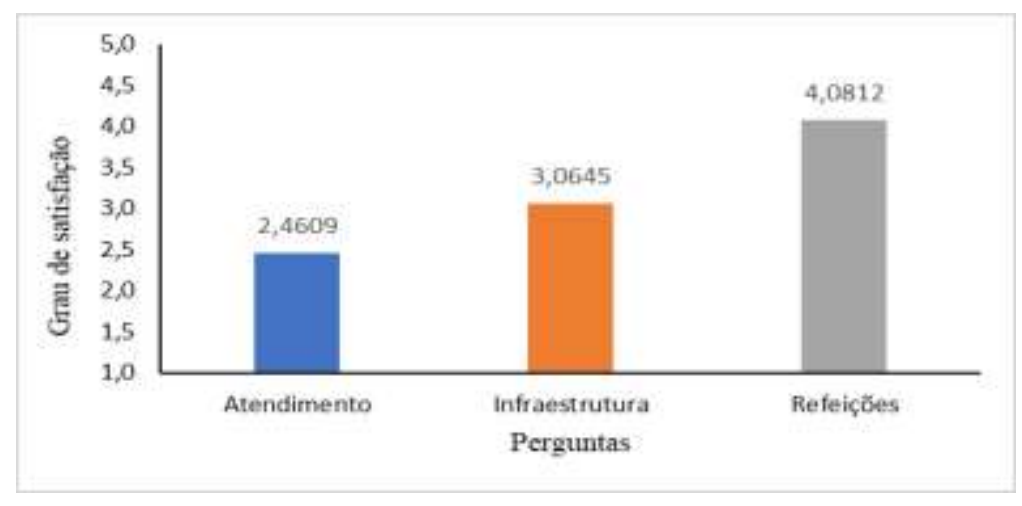

Fonte: Dados da Pesquisa.

\section{Conclusão}

Neste interim, conclui-se que essa pesquisa evidencia a satisfação dos alunos usuários do RU, fornecendo para a equipe gestora do RU informações da prestação de serviços esperados pelos usuários.

A satisfação com as refeições foi o bloco mais bem avaliado pelos alunos usuários, evidenciado a parte primordial de atendimento que é oferecer um alimento de qualidade por restaurantes institucionais.

O bloco infraestrutura apresentou média de satisfação de neutro a concordo parcialmente com a menor satisfação para o estado das cadeiras e maior satisfação para a iluminação do espaço.

O atendimento, foi o bloco de avaliações que teve a menor avaliação com média de 2,4609 sendo o tempo de espera na fila o pior avaliado com média de $(1,6130)$ e o melhor avaliado foi quanto aos funcionários apresentarem uma postura ética no atendimento com média de $(3,8043)$, de uma forma geral o bloco demonstra insatisfação, fato que pode ser evidenciado pela demanda dos alunos que frequentam o RU, por apresentar como evidenciado no bloco refeições, alimentos de qualidade, e oferecer de forma gratuita, no entanto dá sinais de não estar conseguindo acompanhar essa demanda crescente.

A presente pesquisa, apresenta uma limitação que deve ser considerada para estudos, levantamentos de satisfação..., pois ela foi direcionada para alunos de graduação, sendo amostra generalizada para esses alunos, o qual pode ser um equívoco, pois servidores e alunos de pós-graduação possuem avaliações divergentes da dita pesquisa.

Logo sugere-se, para futuros trabalhos a avaliação de satisfação empregada para todos os usuários para os ambientes de alimentação, tanto discentes, docentes e técnicos administrativos, para investigar aspectos relacionados com a convivência social, confraternização, reunião, e se esses itens podem ou não influenciar na satisfação.

\section{Referências}

Abreu, E. S., \& Spinelli, M. G. N. (2009). A unidade de alimentação e nutrição. In: Abreu, E. S., Spinelli, M. G. N., Zanardi, A. M. P. Gestão de unidades de alimentação e nutrição: um modo de fazer. (3a ed.), Metha, 33-40.

Almeida, T. D., Brito neto, J. L., Lakatos, M.; \& Montemor, M. (2008). Relação entre o cardápio do Restaurante Universitário e desperdício. Revista Ciências do Ambiente On-Line. 4(1). 
Alves, M. S., \& Salcedo, D. A. (2014). Indicadores de Qualidade como Instrumento de Gestão: um estudo de caso no Restaurante Universitário da UFPE. In: Encontro de Estudos sobre Tecnologia, Ciência e Gestão da Informação (ENEGI), V, Anais.

Braga, A. C., Pereira, T. L., \& Andrade junior, P. P. (2015). Avaliação de Restaurante Universitário por meio de Indicadores de Qualidade. Desenvolvimento em Questão, Ijuí, 13(30).

Brandão, A. R., \& Giovanoni, A. (2011). Comparação dos cardápios oferecidos em uma unidade de nutrição do município de Teutônia com o programa de alimentação do trabalhador. Revista Destaques Acadêmicos, Lajeado, 3(3). Disponível em: http://univates.br/revistas/index.php/destaques/article/view/116.

Cattafesta, M., Siqueira, J. H., Proenca, L. V. S., Olisosa, P. R., Santos, K. V., Mannato, L., Sabrina, T., \& Molina, M. D. C. B. (2012). Condições higiênicosanitárias de um restaurante universitário e as práticas alimentares de seus usuários. Revista Brasileira de Pesquisa em Saúde, 14, 36-43.

Colares, L. G. T., \& Freitas, C. M. (2007). Processo de trabalho e saúde de trabalhadores de uma Unidade de alimentação e nutrição: entre a prescrição e o real do trabalho. Cad. Saúde Públ., 23, 3011-20. https://doi.org/10.1590/S0102-311X2007001200022.

Carvalho, L. R., Amorim, S. L., \& Tavares M. F. (2003). Sistema de indicadores de qualidade como ferramenta de gestão em um restaurante universitário: estudo de caso. In: Encontro Nacional de Engenharia de Produção, XXIII, Anais.

Dipietro, R. B., \& Levitt, J. (2017). Restaurant Authenticity: Factors That Influence Perception, Satisfaction and Return Intentions at Regional AmericanStyle Restaurants. International Journal of Hospitality \& Tourism Administration.

Domingues, O., \& Gouvêa, M. A. (2012). Qualidade percebida em atividades de gerenciamento por categoria em supermercados. Revista de Gestão; 19(4): 589-608.

Gasparin, E. et al. (2012). Perfil nutricional e percepção de conforto dos comensais do Restaurante Universitário da UTFPR - Campus Medianeira. In: Seminário de Iniciação Científica e Tecnológica da UTFPR, XVII, Anais..., Curitiba, PR, Brasil, 07 e 08 de novembro de 2012.

Gardin, E. T. O., \& Cruvinel, E. B. S. (2014). Avaliação da satisfação dos clientes do restaurante universitário (RU) do Campus Londrina da Universidade Tecnológica Federal do Paraná. Trabalho de Conclusão de Curso. Curso Superior de Tecnologia em Alimentos. Universidade Tecnológica Federal do Paraná - UTFPR, Londrina.

Härter, A. C., Silva, C. E. S. F., Sneyder, D., \& Siqueira, R. F. (2013). Estudo de Caso Sobre a Satisfação dos usuários do Restaurante Universitário-RU. Revista de Estudos Sociais, Cuiabá, 15 (30).

Jacob, A. V. et al. (2006). Avaliação da satisfação dos clientes e diagnóstico de filas no Restaurante Universitário Noturno da UFSC. In: Semana de Engenharia de Produção Sul-Americana, VI, Anais..., Florianópolis, SC, Brasil, novembro de 2006.

Junior, F. J. M. et al., (2015). Satisfação dos usuários do Restaurante Universitário da Universidade Federal de Santa Maria: Uma análise descritiva. Revista Sociais e Humanas, Santa Maria. 28(2). 10.5902/ 2317175814891, disponível em: https://periodicos.ufsm.br/sociaisehumanas/article/view/14891.

Lira, C. R. N., Carmo, A. F. F., Brandão, T. M., \& Fonseca, M. C. P. (2020) Variáveis relacionadas à satisfação de estudantes com o serviço e cardápio ofertado em Restaurante Universitário. Braz. J. of Develop., 6(8), 56617 - 56628

Longart, P. (2015). Consumer Decision Making in Restaurant Selection. Unpublished PhD Thesis. Coventry: Coventry University Buckinghamshire New University.

Malhotra, N. (2012). Pesquisa de marketing: uma orientação aplicada. (6a ed.), Editora Bookman.

Maia, T. M. L. (2008). Planejamento e Gestão Estratégica para o Restaurante Universitário da UFC em um cenário de expansão do número de alunos. (Dissertação) 106f. Fortaleza, CE. Programa de Pós-Graduação em Políticas Públicas e Gestão da Educação Superior, Universidade Federal do Ceará.

Marques, C. S., Pereira, B. A. D., \& Alves, J. N. (2010). Identificação dos principais fatores relacionados à infraestrutura universitária: uma análise em uma IES pública. Revista Sociais e Humanas, Santa Maria, 23(01), p. 91-103.

Mattar, F. N. (2001). Pesquisa de marketing: edição compacta. Atlas.

Medeiros, L. G. F., Mello, L. T. C., Araújo. I. D. L., \& Bulhões, L. A. (2013). A gestão de processos como fonte de melhorias: aplicação de técnicas de mapeamento de processos em um Restaurante Universitário. In: Congresso Nacional de Excelência em Gestão (CNEG), IX, Anais..., 2013.

Milet, E. B. (1997). Qualidade em serviços: princípios para a gestão contemporânea das organizações. Ediouro.

Minayo, M. C. S. (1994). Ciência, técnica e arte: o desafio da pesquisa social. In: Minayo, M. C. S. (org.). Pesquisa social: teoria, método e criatividade. 22. ed. Vozes.

Motta, P. C. (1999). Serviços: pesquisando a satisfação do consumidor. (3a ed.), Imprinta Express.

Moura, M. A. P. et al. (2015). Grau de satisfação dos alunos da Universidade Federal do Piauí em relação aos serviços prestados pelo Restaurante Universitário do Campus Ministro Reis Velloso, em Parnaíba - PI. In: Congresso Internacional de Administração, Anais..., Ponta Grossa, PR, Brasil, 22 a 26 de setembro de 2015 .

Oliveira, N. M. C. (2013). Simulação a eventos discretos para a redução do tempo de espera em um restaurante (Dissertação).126 f. Itajubá, MG. Programa de Pós-graduação em Engenharia de Produção. Universidade Federal de Itajubá.

Oliveira, C. S., \& Alves, F. S. (2009). Educação nutricional em unidade de nutrição, direcionada para consumo de pratos protéicos um estudo de caso. Alim. Nutr., 19(4), 435-440. 
Research, Society and Development, v. 11, n. 2, e14011225557, 2022

(CC BY 4.0) | ISSN 2525-3409 | DOI: http://dx.doi.org/10.33448/rsd-v11i2.25557

Oliveira, Z. M. C. (2004). A Unidade de alimentação e nutrição na empresa. In: Teixeira S. M. F. G. et al. Administração aplicada às unidades de alimentação e nutrição. Atheneu, 13-77.

Oliver, R. L. (1997). Satisfaction: a behavioral approach. McGraw-Hill.

Proença, R. P. C., Souza, A. A., \& Hering, B. (2005). Qualidade nutricional e sensorial na produção de refeiçães. Florianópolis: EdUFSC, 221p.

Proença, R.P.C. (1999). Inovações tecnológicas na produção de refeições: conceitos e aplicações básicas. Higiene Alimentar, 13(63), 24-30.

Parasuraman, A., Zeithaml, V. A., \& Berry, L. L. (1985). A conceptual model of service quality and its implications for future research. Journal of Marketing ;49(4): 41-50. 10.2307/1251430.

Passador, J. L., Filho, D. O. L., Spanhol, P. S., Rodrigues, F. S., \& Sabes, J. J. S. (2006) A percepção do consumidor de alimentos "fora de casa": um estudo multicaso na cidade de Campo Grande/MS.In: SIMPEP, XIII, Anais..., Brasil, 6 a 8 de Novembro de 2006.

Rebelato, M. G. (1997) Uma análise sobre a estratégia competitiva e operacional dos restaurantes self-service. Gest. Prod. 4(3), 321-334. https://doi.org/101590/S0104-530X1997000300006.

Reis, I. (2001). Escala SERVQUAL modificada: avaliação da qualidade percebida do serviço de lazer oferecido por um complexo poliesportivo num parque florestal. Universidade Federal de Santa Catarina (UFSC).

Rohr, A. R., Masiero, M. S., \& Kliemann neto, F. J. (2010). Proposta de um sistema de gestão de custos para o Restaurante Universitário da Universidade federal do Rio Grande do Sul. In: Encontro Nacional de Engenharia de Produção, XXX, Anais.

Rossi, C. A. V., \& Slongo, L. A. (1998). Pesquisa de Satisfação de clientes: o Estado-da-Arte e Preposição de um Método Brasileiro. Revista de Administração Contemporânea, 2(1).

Santos, B.A.D.R., \& Vera, L.A.R. (2021) Avaliação da qualidade dos serviços do restaurante universitário da Universidade Federal da Bahia na percepção dos usuários. Marketing \& Tourism Review, 5(2). https://doi.org/10.29149/mtr.v5i2.5943

Saraiva, M. (2016). The Degree of Customer Satisfaction in University Restaurants: The Case of the University of Evora in Portugal. Edulearn11: 3rd International Conference On Education And New Learning Technologies (2011):1597-1605. Web. 129 Acta de Ciências e Saúde, 2(5).

Solomon, M. R. (2002). O comportamento do consumidor: Comprando, possuindo e sendo. (5a ed.), Bookman.

Trierweiller, A. C., Weise., A. D., Perreira, V. L. D. V., Junior, W. P., \& Rocha, A. R. (2011). Diagnóstico de satisfação de clientes como ferramenta para fidelização: um estudo de caso em cinema cult. Revista de Administração da UNIMEP. 9(1).

Vieira, V.G., \& Cavalcanti, E.P. (2020) Análise dos serviços de um restaurante universitário na perspectiva dos usuários. Research, Society and Development, 9(10), e4519108715 http://dx.doi.org/10.33448/rsd-v9i10.8715. 